\title{
Fuerza explosiva en adultas mayores, efectos del entrenamiento en fuerza máxima Explosive strength in older adults, training effects on maximum strength
}

*Laura Elizabeth Castro Jiménez, *Angela Yazmin Gálvez Pardo, *Gustavo Andrés Guzmán Quintero, *Ana Isabel Garcia Muñoz *Universidad Santo Tomás (Colombia), ** Escuela Militar de Cadetes General José María Córdova (Colombia)

Resumen: Introducción. En los adultos mayores existe una disminución de la fuerza muscular que es más significativa en mujeres que en los hombres. Objetivo. Conocer los cambios que en fuerza explosiva puedan generarse, posterior a la intervención con un programa de entrenamiento en fuerza máxima. Materiales y Métodos. Estudio cuasiexperimental con diseño pre-prueba y post-prueba, en una cohorte de adultas mayores quienes durante 12 semanas, recibieron entrenamiento en fuerza máxima, entre un 44\% y un 54\% de su RM. Los criterios de inclusión fueron, ser adultas mayores (e»60 años), con índice de masa corporal (IMC: $19-30 \mathrm{~kg} / \mathrm{m}^{2}$ ), que asistieran al programa de actividad física para el adulto mayor, de la Universidad Santo Tomás en Bogotá (Colombia). Resultados. Se encontraron cambios en la fuerza explosiva con el entrenamiento, pero no son estadísticamente significativos, pero en cambio se encontraron cambios en la antropometría que si tienen una correlación significativa con la fuerza explosiva medida a través de SQJ. Conclusiones. Los resultados evidencian que es posible generar cambios en porcentajes de tejido graso a través de entrenamiento en fuerza y no exclusivamente en resistencia aeróbica y es necesario aumentar la muestra poblacional para reconocer con mayor claridad los cambios que se puedan generar en fuerza en el adulto mayor.

Palabras clave: Fuerza Muscular, Educación y Entrenamiento Físico, adulto mayor, mujeres.

Abstract. Introduction. In older adults there is a decrease in muscle strength that is more significant in women than in men. Objective. Assess the changes in explosive force that can be generated after an intervention with a training program on maximum force. Materials and methods. A quasiexperimental study with pre-test and post-test design, in a cohort of older adults who, during 12 weeks, received training on maximum strength, between $44 \%$ and $54 \%$ of their MRI. The inclusion criteria were to be older adults (e»60 years);to have a body mass index of $19-30 \mathrm{~kg} / \mathrm{m} 2$ ); to attend the physical activity program for the elderly of the Santo Tomás University in Bogotá (Colombia). Results. We found changes in the explosive force after the training; however, they are not statistically significant. Changes were found in anthropometry, this showing significant correlation with explosive force measured through SQJ. Conclusions. The results show that it is possible to generate changes in percentages of fat tissue through strength training and not exclusively with aerobic resistance. Also, it is necessary to increase the sample size in order to recognize with greater clarity the changes that can be generated in strength in elderly.

Key words: Muscle Strength, Physical Education and Training, Aged, women.

\section{Introducción}

A medida que transcurre la vida del ser humano se van presentando una serie de cambios en las capacidades físicas, es así como las modificaciones del sistema locomotor, tales como la disminución de la fuerza, de la movilidad, de la resistencia y del equilibrio (Zillikens, et al., 2017; Guillen, Bueno, Gutiérrez \& Guerra, 2018), sumadas a la presencia de enfermedades cardiovasculares, metabólicas y endocrinas, limitan la capacidad del adulto mayor para ejecutar actividades de la vida diaria como caminar, levantarse y tomar objetos, incrementando con ello el riesgo de caídas y la posibilidad de afectar su calidad de vida (Ramírez \& León, 2012; Salazar, Ramírez, Chaparro \& León, 2014). Se ha sostenido que estas modificaciones se presentan principalmente como consecuencia de la pérdida de tejido muscular, pero recientemente se ha encontrado una asociación con el deterioro de la función neuromuscular (Laroche, Knight, Dickie, Lussier \& Roy, 2007).

Si a esta situación se suma el tiempo sedentario, definido como cualquier comportamiento en posición sentada o reclinada, con bajo gasto energético, es decir $\leq 1,5$ equivalentes metabólicos de la tarea (METS), el riesgo incrementa. $\mathrm{Al}$ respecto se ha documentado una relación directamente proporcional entre la suma del tiempo sedentario y la disminución de la fuerza de agarre y potencia de las piernas, siendo mayor esta relación en mujeres (Copeland, et al., 2017; Del Valle, Prieto, Nistal, Martínez \& Ruiz, 2014). Ésta potencia o fuerza explosiva de miembros inferiores tiende a disminuir progresivamente y en mayor porcentaje que la fuerza muscular máxima (Edholh, Strandberg \& Kadi, 2017), constituyéndose en uno de los predictores más fuertes del estado funcional, del adulto mayor (Foldvari et al., 2000).

La fuerza explosiva ha sido definida, desde la mirada de Juárez et al. (2008) como la relación entre la fuerza producida y el tiempo necesario para generarla. Los mismos autores sostienen que para su optimización, es necesario potenciar la fuerza dinámica máxima, que sería en sus propias palabras: «la expresión máxima de fuerza cuando la resistencia

Fecha recepción: 13-07-18. Fecha de aceptación: 21-12-18

Laura Elizabeth Castro jimenez

laura.castro@usantotomas.edu.co sólo se puede desplazar una vez, o se desplaza ligeramente o transcurre a muy baja velocidad en una fase del movimiento» (p.2).

Dentro de los métodos empleados para valorar la fuerza explosiva se ha descrito el Squat Jump (SJ) o salto «de parado»: de la batería de Bosco, el cual consiste en realizar un salto vertical sin contra-movimiento, partiendo de la posición de medio Squat (rodilla flexionada a $90^{\circ}$ aproximadamente), con el tronco recto y las manos en la cadera. En este salto solo se ejecuta un ciclo de movimiento de acortamiento muscular (Bobbert \& Soest, 1994).

El entrenamiento de fuerza puede mejorar la fuerza muscular en sí misma, la resistencia y mantener la masa magra, optimizando con ello, la capacidad de las personas mayores para realizar actividades de la vida diaria. Adicionalmente el entrenamiento en fuerza ha documentado cambios en la composición corporal, flexibilidad y un incremento en las fibras tipo II b (Ramírez \& León, 2012), así como en la autoconfianza y percepción de la capacidad para caminar del adulto mayor (Vidarte, Quintero \& Herazo, 2012; García \& Froment, 2018), siendo el entrenamiento en fuerza explosiva, el que ejerce mayor influencia en el rendimiento físico y en la disminución del riesgo de caídas, en esta población. Además, la dinámica del ejercicio físico y nuevas tendencias se obtiene beneficios de carácter social, individual y emocional que complementan el ideal de bi-corporiedad (Keogh, Kilding, Pidgeon, Ashley \& Gillis, 2009; Vaquero-Cristóbol, Martínez, Alacid y Ros, 2015; Villareal, Moncada, Gallegos \& Ruiz, 2016). Específicamente en Colombia se evidenció con 16 semanas de entrenamiento en cualidades físicas, potencia aeróbica, flexibilidad y fuerza, una mejoría significativa de la fuerza dinámica, un cambio de niveles de fuerza estática y mejoría en la capacidad cardiorrespiratoria (Sandoval, Camargo, González \& Vélez, 2007).

Desde esta panorámica descrita y teniendo en cuenta que los adultos mayores tienen la misma capacidad para mejorar la fuerza que sujetos más jóvenes (Frischknecht, 1998; Knight \& Kamen, 2001) y que las discapacidades funcionales relacionadas con la edad, son más comunes entre las mujeres (Edholm, Strandberg \& Kadi, 2017), se planteó ésta investigación, con el objetivo de evidenciar los cambios, que en fuerza explosiva puedan generarse, posterior a la intervención con un programa de entrenamiento en fuerza máxima, toda vez que 
según Copeland y su grupo de trabajo, aún faltan de estudios que evalúen apropiadamente el impacto de estas intervenciones sobre los resultados de salud en los sujetos geriátricos (Copeland et al., 2017).

\section{Materiales y Métodos}

Estudio cuasiexperimental con diseño pre-prueba y post-prueba, en una cohorte de adultas mayores quienes durante 12 semanas, recibieron entrenamiento en fuerza máxima. La población estuvo conformada por 80 mujeres y la muestra por 27. El tamaño muestral se determinó a través de diferencias de medias, en el que se tuvo como referencia un intervalo de confianza del 97\%, con un margen de error del 0,03 (Gama \& Gómez-Conesa, 2008; Vidarte et al., 2012). Como criterios de inclusión se contempló, ser adultas mayores ( $\geq 60$ años), con índice de masa corporal (IMC: $19-30 \mathrm{~kg} / \mathrm{m}^{2}$ ), que asistieran al programa de actividad física para el adulto mayor, de la Universidad Santo Tomás en Bogotá (Colombia). Se excluyeron aquellas participantes con Hipertensión arterial no controlada, Enfermedad pulmonar crónica agudizada, enfermedades metabólicas descompensadas (Diabetes tipo I y II), enfermedades reumatológicas, musculoesqueléticas como la artrosis y enfermedades psiquiátricas. Así mismo, aquellas adultas que se encontraran recibiendo suplencia nutricional o dieta específica, fueran fumadoras activas (Mínimo dos años antes de la investigación), con incapacidad autoinformada para caminar, que refirieran ingesta mayor a 2 tragos día de bebidas alcohólicas y/o con contraindicaciones médicas al ejercicio regular. Esta información se corroboró con la historia clínica.

Todas las participantes firmaron consentimiento informado, aprobado por el comité de ética de la universidad en mención, con código A002-16062016. Los instrumentos fueron evaluados a través de validez de contenido y con juicio de expertos en procesos de evaluación en adultos mayores.

\section{Protocolos de evaluación}

Para la evaluación de la fuerza máxima, se diseñó y aplicó el siguiente protocolo: La fuerza máxima se obtuvo a partir del método de 1RM (una repetición) en el movimiento de extensión, de miembros inferiores, en la maquina Leg Press, partiendo de posición sentada con un ángulo de flexión de rodilla de $90^{\circ}$. Las adultas mayores realizaron la extensión a una velocidad lenta sin llegar a tener una extensión completa $\left(180^{\circ}\right)$. Previo, se realizó un calentamiento de movilidad articular y en bicicleta de 5 minutos respectivamente. Luego de esto, se realizó, directamente en la máquina, otro calentamiento de 15 repeticiones con cargas del $20 \%$ de la escala de percepción del esfuerzo OMNI-RES (Robertson et al., 2003). En este calentamiento se hizo énfasis en la técnica de ejecución. Para determinar 1RM, se incrementó la carga progresivamente en un 10\% con intervalos de tres minutos de recuperación, de acuerdo con los resultados de la escala OMNI-RES, hasta que la participante fuera incapaz de realizar una repetición completa. La carga más alta movilizada cumpliendo esta condición, fue la considerada como la fuerza máxima. Se empleó el SQUAT JUMP para medir la fuerza explosiva con el OptoGait (MD number 467316/R) (Izquierdo, Aguado, González, Lopez \& Häkkinen, 1999).

\section{Programa de entrenamiento}

El entrenamiento de fuerza se realizó durante un periodo de 12 semanas con una frecuencia de tres veces por semana. Realizando un entrenamiento entre un $44 \%$ y un $54 \%$ de su RM obtenido en dicha prueba, de acuerdo con el protocolo diseñado para tal fin (evaluación a través de RM sub-máxima). Se trabajó en la maquina Leg Press, tres series de 10 repeticiones, con intervalo de recuperación de 1 minuto entre cada serie. Cada cuatro semanas se incrementó en un $10 \%$ la carga.

Como variables a analizar se incluyó el peso, el IMC, el porcentaje graso total y visceral, así como el porcentaje muscular, en relación con la fuerza explosiva. La toma de datos se hizo en tres momentos, al iniciar el programa de entrenamiento; en la mitad del tiempo de entrenamiento (6 semanas) y en la última semana de entrenamiento.

Para el análisis estadístico se empleó la prueba no paramétrica de
Kolmogorov- Smirnov, para determinar normalidad de los datos. Para las correlaciones se empleó el coeficiente de Pearson, con un intervalo de confianza del 95\% y una significancia < .05, utilizando el programa estadístico de SPSS versión 22.

\section{Resultados}

Se evaluó una población total de 27 mujeres; con edad media de 63 años (DS $\pm 3,6$ ), encontrando una media pre-entrenamiento del peso corporal en 62,2 kg. (DS $\pm 10,6$ ), porcentaje graso 38,9 \% (DS $\pm 6,1$ ), porcentaje de grasa visceral de $8,6 \%$ (DS $\pm 2,7$ ) y el porcentaje muscular de 24,9 \% (DS $\pm 2,1$ ). Los valores post-entrenamiento del porcentaje graso fueron $38,7 \%(\mathrm{DS} \pm 6,05)$. El porcentaje de grasa visceral fue $8,8 \%$ (DS $\pm 2,1$ ), el porcentaje muscular presento valores medios de $24,9 \%$ (DS $\pm 2,07)$. Se evaluó también la talla media de las adultas mayores, la cual fue de $153,14 \mathrm{~cm}$. (DS $\pm 3,6$ ), partiendo de la relación talla y peso se obtuvo un IMC pre-entrenamiento de $26,5 \mathrm{Kg}$. $/ \mathrm{m}^{2}(\mathrm{DS} \pm 4,04)$ y IMC post-entrenamiento 26,1 Kg. $/ \mathrm{m}^{2}$ (DS $\pm 3,8$ ).

En la prueba de salto, se encontró una media pre-entrenamiento de 11,4 Julios (DS $\pm 2,7$ ) Vs. 10,9 Julios (DS $\pm 2,8$ ), post entrenamiento, sin diferencia, estadísticamente significativa (valor de $\mathrm{p}=.456$ ) (Tabla 1).

Tabla 1

Correlación entre SQUAT JUMP y Variables Antropométricas

\begin{tabular}{|c|c|c|c|}
\hline & & Squat Juamp pretest & t Squat Juamp Postest \\
\hline \multirow{3}{*}{ Peso primera toma } & Correlación de Pearson &,- 112 &,$- 471^{*}$ \\
\hline & Sig. (bilateral) & 603 & 015 \\
\hline & $\mathrm{N}$ & 27 & 27 \\
\hline \multirow{3}{*}{$\%$ Graso primera toma } & Correlación de Pearson &,- 358 &,$- 629 * *$ \\
\hline & Sig. (bilateral) & 094 & ,001 \\
\hline & $\mathrm{N}$ & 27 & 27 \\
\hline \multirow{3}{*}{ \% Muscular primera toma } & Correlación de Pearson & $488^{*}$ & $606 * *$ \\
\hline & Sig. (bilateral) &, 018 & 001 \\
\hline & $\mathrm{N}$ & 27 & 27 \\
\hline \multirow{3}{*}{$\%$ Visceral primera toma } & Correlación de Pearson &,$- 505^{*}$ &,$- 459^{*}$ \\
\hline & Sig. (bilateral) &, 014 &, 021 \\
\hline & $\mathrm{N}$ & 27 & 27 \\
\hline \multirow{3}{*}{ Imc primera toma } & Correlación de Pearson &,- 331 &,$- 551 * *$ \\
\hline & Sig. (bilateral) &, 114 & ,004 \\
\hline & $\mathrm{N} \cdot(0)$ & 27 & 27 \\
\hline \multirow{3}{*}{ Peso segunda toma } & Correlación de Pearson &,- 129 &,$- 504 * *$ \\
\hline & Sig. (bilateral) & ,549 & ,009 \\
\hline & $\mathrm{N}$ & 27 & 27 \\
\hline \multirow{3}{*}{ Masa grasa tercera toma } & Correlación de Pearson &,$- 432^{*}$ &,$- 619 * *$ \\
\hline & Sig. (bilateral) & 035 & ,001 \\
\hline & 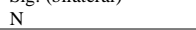 & 27 & 27 \\
\hline \multirow{3}{*}{$\begin{array}{l}\text { Masa grasa visceral segunda } \\
\text { toma }\end{array}$} & Correlación de Pearson &,$- 448^{*}$ &,$- 664^{* *}$ \\
\hline & Sig. (bilateral) &, 028 &, 000 \\
\hline & $\mathrm{N}$ & 27 & 27 \\
\hline \multirow{3}{*}{ IMC segunda toma } & Correlación de Pearson &,- 348 &,$- 589 * *$ \\
\hline & Sig. (bilateral) & 095 & ,002 \\
\hline & $\mathrm{N}$. (nitictit) & 27 & 27 \\
\hline \multirow{3}{*}{ Masa muscular segunda toma } & Correlación de Pearson &, $584^{* *}$ &, $533 * *$ \\
\hline & Sig. (bilateral) & ,003 & ,005 \\
\hline & $\mathrm{N} \cdot(\mathrm{min})$ & 27 & 27 \\
\hline
\end{tabular}

. Lacol

**. La correlación es sign

Así mismo, los factores antropométricos presentaron una incidencia sobre la capacidad de salto, encontrándose a nivel del peso corporal una correlación negativa y estadísticamente significativa ( $\mathrm{r}=-.129$ Vs. $r=-.504)$, posterior al entrenamiento. Con respecto a los componentes del peso corporal se encontró una correlación negativa, es decir, los valores de correlación del porcentaje graso pre-test fue de -.432 y paso a -.619 post-test aumentando su significancia de $\mathrm{p}=.05$ a .01 . Así mismo, la grasa visceral con valores de correlación pre-test de -.448 pasó a -.664 post-test con una correlación significativa de $\mathrm{p}=.01$. Por ende, el programa de entrenamiento en fuerza muscular logró cambios directos en la composición corporal y estas variaciones a su vez presentaron correlaciones significativa con los resultados de las pruebas de fuerza explosivas evaluadas a través de SJ, evidenciándose que aquellas adultas con incremento en el porcentaje de masa muscular y en el porcentaje de grasa, presentaron correlación positiva en el primer caso $(\mathrm{r}=.488$ Vs. $\mathrm{r}=$.606) y en el segundo caso correlación negativa $(\mathrm{r}=-.357$ Vs. - .629), con respecto al rendimiento en el salto.

Complementario a esto, la masa muscular total, también se correlacionó con el rendimiento en la prueba ( $\mathrm{r}=.584 \mathrm{Vs}$. $\mathrm{r}=.533)$, mientras que el IMC se correlacionó negativamente (-.348a - .589) con el rendimiento en el salto vertical, desde el inicio del entrenamiento. Por 
su parte, la grasa visceral también se correlacionó negativamente con el rendimiento del salto.

\section{Discusión}

Los resultados encontrados evidencian que no hubo un aumento estadísticamente significativo, en la fuerza explosiva (media pre de 11,41 Julios DS $\pm 2,69$ y post 10,91 DS $\pm 2,79$ ), posterior al entrenamiento en fuerza máxima, pudiendo deberse éste hallazgo, en primera instancia a que los aspectos coordinativos en la población estudiada fueron bajos, haciendo que biomecánicamente, las cadenas cinéticas musculares no fueran funcionales a las pruebas de salto puesto que no hubo una diferenciación en las fases que trae el movimiento. En este sentido, Stengel et al., (2005) menciona que la coordinación permite economizar la acción motriz, siendo indispensable para movimientos que requieran velocidad y eficacia; a su vez es vista como un engranaje de las capacidades condicionales de fuerza, resistencia y velocidad (estas últimas evaluadas en el SJ). La coordinación, presenta un componente de reacción «capacidad de reacción» relacionándolo con la capacidad de poder realizar una acción motriz en un corto lapso de tiempo ante una reacción externa; como se mencionó anteriormente la diferenciación es otro componente de la coordinación que permite tener un ajuste entre las fases de una acción motriz y de la musculatura que va a ser implicada (Kemmler et al., 2005; Stengel et al., 2005). Al respecto, el estudio realizado por Hughes et al., (2001), mostró que un entrenamiento de fuerza de 10 semanas logró aumentar la fuerza en un $60 \%$ y esta ganancia se asoció con una mejor coordinación en la marcha, así como con el aumento de la velocidad y condición propioceptiva en los gestos de la vida diaria.

En segundo lugar, la no mejora de la potencia de salto, pude deberse que no se tuvo en cuenta si las participantes presentaban algún déficit nutricional, lo cual pudo interferir en los resultados. Al respecto, Edholm et al., (2017) evidenciaron que el entrenamiento de la fuerza, asociado a una dieta saludable rica en ácidos grasos polinsaturados n-3 (PUFA n3), mejora, entre otros, la fuerza dinámica explosiva durante movimientos aislados de extremidades inferiores.

En la revisión bibliográfica a nivel internacional y nacional no se encontraron, estudios semejantes donde la fuerza máxima fuese el medio de entrenamiento a la fuerza explosiva. No obstante Ramírez, Da Silva y Lancho (2007) aplicaron un entrenamiento de 22 semanas, con la diferenciación de un trabajo de fuerza mediado por saltos verticales y horizontales (entrenamiento de miembros inferiores a través de pliométricos), este grupo de investigadores manejaron 3 grupos (de control masculino, experimental masculino y experimental femenino); reportando en sus resultados a nivel de fuerza explosiva (valores significativos $\mathrm{p}<.05$ ) particularmente se observó un cambio notorio de contramovimiento (CMJ), contramovimiento con balanceo de brazos (CMJas) y Squat jump (SJ); respecto a lo anterior, se puede establecer que existen métodos de entrenamiento con mayor funcionalidad; además de esto, la población a trabajar presentaba prácticas en gimnasia lo que muestra que su capacidad coordinativa, manejo de técnica, donde la huella motora es un factor incidente sobre la mejora, por lo tanto, lo establecido anteriormente puede tener un valor representativo en este estudio (Ramírez et al., 2007; Barbosa, Rodríguez et al., 2007).

El porcentaje de masa muscular mostró correlación positiva desde el inicio hasta el final, con la potencia de salto, lo que es de esperarse pues a mayor desarrollo de fibras musculares, mayor desarrollo de la fuerza existirá y por tanto menor probabilidad de disfunción de cualidades físicas (Hall et al., 2017). En tal sentido, al observarse mejoría en la masa muscular, es posible inferir que el entrenamiento de la fuerza máxima, en adultas mayores puede disminuir, el riesgo de padecer enfermedades crónicas, principalmente las cardiovasculares y al mismo tiempo optimizar la fuerza isocinética de Cuádriceps e isquiotibiales (Sanchez, 2009; Araya et al., 2012).

Con respecto a la masa grasa y el volumen de grasa visceral, con los cuales se observó una correlación negativa con la potencia de salto, algunos trabajos en deportistas han documentado que cuanto más alta es la cantidad de masa grasa, menor es el rendimiento del salto vertical, sobre todo si la misma se acumula en Miembros inferiores o en el abdomen (Pliegue de muslo y cintura) (Rendón et al., 2017). Una publicación reciente, menciona que, en las adultas mayores existe una mayor predisposición a la pérdida de masa muscular con respecto a los hombres y que dicha pérdida se da principalmente en miembros inferiores, lo que podría explicar en el caso del presento estudio, la correlación hallada y al mismo tiempo ser un factor determinante de los resultados encontrados, pues el pliegue del muslo no fue medido y podría ser un factor de confusión (Brady \& Straight, 2014).

Vale la pena mencionar que, a nivel de composición corporal, como consecuencia del trabajo de fuerza máxima; se obtuvo mejoras y evidenciaron correlaciones altamente significativas con estimaciones de $<<.01$ y .05, sobre los cambios de composición corporal y los cambios en los resultados de la prueba de salto. Dichas evidencias, fueron encontradas en el peso corporal, porcentaje graso y porcentaje muscular; en donde se dice que tanto el peso como el componente graso (a su vez el visceral) presentan una correlación negativa y el componente muscular correlación positiva respecto a la capacidad de salto (Ramírez et al., 2007).

El panorama, en cuanto a la mejora de la composición corporal, Ramírez y León (2012) trabajan con fuerza explosiva correlacionándola a otras capacidades (funcionalidad, agilidad etc.); se evaluó una población total de 27 mujeres; de una edad media de 63 años (DS $\pm 3,641$ ). Se hicieron toma de datos antropométricos, haciéndose registros pre y post entrenamiento; encontrando una media pre-entrenamiento del peso corporal en 62,219 kg. (DS $\pm 10,6161$ ), porcentaje graso 38,923\% (DS $\pm 6,1155$ ), porcentaje graso visceral de $8,615 \%$ (DS $\pm 2,7288$ ) y el porcentaje muscular de $24,973 \%$ (DS $\pm 2,1514$ ). Los valores postentrenamiento del porcentaje graso fueron $38,7926 \%$ (DS $\pm 6,05703$ ); el porcentaje graso visceral de 8,8111 \% (DS $\pm 2,1514$ ), el porcentaje muscular presento valores medios de 24,9\% (DS $\pm 2,07$ ). Se evaluó también la talla media de las adultas mayores, la cual fue de 153,141 cm. (DS $\pm 3,641$ ), partiendo de la relación talla y peso; se obtuvo un IMC pre- entrenamiento de $26,5622 \mathrm{Kg}$. $/ \mathrm{m}^{2}$ (DS $\pm 4,04381$ ) e IMC postentrenamiento $26,1 \mathrm{Kg} . / \mathrm{m}^{2}$ (DS $\left.\pm 3,8631\right)$. Los datos antropométricos encontrados en este estudio son semejantes en peso corporal y IMC respecto a la media estadística de nuestro estudio; en comparación de \% muscular y \% graso difieren sustancialmente; la población trabajada en el presente estudio presenta niveles más bajos y se observaron cambios en la investigación de Ramírez et al., (2012)no se encontraron cambios significativos.

De modo similar, Ramírez et al., (2007), presenta valores de composición corporal sin correlacionarlos con la capacidad de salto; cabe recalcar que este estudio evidencia los cambios obtenidos tras la intervención de entrenamiento. Encontrándose valores (grupo de control masculino y grupo de control femenino) pre Peso: 79,3kg y $73 \mathrm{~kg}$. (DS $\pm 8,4$ y 10,8), masa grasa 23,9\% y $27,8 \%$ (DS $\pm 1,7$ y 0,9), IMC $28,1 \mathrm{Kg}-/ \mathrm{m}^{2}$ y $31,3 \mathrm{Kg} . / \mathrm{m}^{2}$ (DS $\pm 2,5$ y 4,4 ) y post $79,7 \mathrm{Kg} . \mathrm{m}^{2}$ (DS $\pm 6,4$ y 9,6), masa grasa 23,4\%y 26,1\% (DS $\pm 1,3$ y 3,9) y IMC 28,9 $\mathrm{Kg} . / \mathrm{m}^{2}$ y 32,1 Kg. $/ \mathrm{m}^{2}$ (DS $\pm 2,1$ y 2,2) donde determinaron que solo los datos obtenidos en el grupo de control masculino fueron significativos; lo que permite fortalecer la idea de funcionalidad de dicho trabajo.

Respecto a los resultados de dicho estudio, se puede concluir que posiblemente para que haya una mejora en la capacidad de salto es necesario modificar en primera medida los componentes antropométricos; sumado a esto a nivel metodológico se debe buscar un proceso de enseñanza respecto al gesto de las pruebas puesto que como se evidencio durante estas la población a estudiar evidenciaba una necesidad de mejora en componentes de diferenciación de secuencias de movimiento lo que posiblemente puede llevar a que la activación muscular no corresponda a la necesaria durante la prueba de salto(SJ). Otra alternativa está en evaluar con relación a la funcionalidad de sujetos acorde puesto que el objetivo del ejercicio físico para la salud en estas edades se vincula a la calidad de vida como lo evidencia Enriquez-Reina, Carranza \& Navarro (2019) quienes implementaron la batería corta de desempeño físico y obtuvieron una caracterización óptima de la población por principio de individualidad.

Son diversas las dudas que se presentan sobre las metodologías a 
aplicar, las pruebas de control, la periodización, los contenidos empleados y las intensidades de carga que deben usarse. De los ensayos de fuerza aplicados con sujetos longevos, destaca por sus resultados el entrenamiento progresivo de la fuerza máxima combinado con ejercicios de ejecución rápida, con el objetivo de mejorar la fuerza máxima y la fuerza explosiva. Otra alternativa para mejorar la fuerza explosiva podría ser el empleo de saltos para valorar y entrenar la potencia muscular en diferentemedio como por ejemplo en agua(Medina, Padilla, Rodríguez \& Alejo, 2018), que al no valerse de máquinas que condicionen la velocidad del movimiento y facilitar la graduación consciente del ángulo articular, se asemejan más a los gestos técnicos y a la actividad funcional innata del ser humano.

Partiendo de lo anterior, no solo las nuevas alternativas facilitan la metodología del entrenamiento sino también se vincula al beneficio de las capacidades de las personas longevas; donde alternativas como la hidrogenásis presenta cambios significativos en las capacidades físicas condicionales (resistencia aeróbica, resistencia muscular, agilidad y equilibrio dinámico; esto es expresado indicando interacción significativa ( $\mathrm{p}=.052$ ) entre grupos de control (10 Adultas mayores) y experimental (16 adultas mayores) en las mediciones de la prueba de resistencia a la fuerza en extremidades inferiores, la prueba de agilidad de 2.4 metros tuvo interacción significativa $(\leq .01)$ entre grupos y mediciones, las pruebas de capacidad aeróbica step test de 2 minutos ( $\mathrm{p}=.02$ ) y caminar 6 minutos indicaron interacción significativa entre grupos y mediciones ( $p=.50)$; Se infiere que la hidrogimnasia en las adultas mayores participantes es eficaz en la mejora de las capacidades físicas principalmente aeróbicas, de resistencia a la fuerza y agilidad de miembros inferiores (Hall et al., 2014).

\section{Conclusiones}

Este tipo de investigaciones deben ampliar en el futuro la muestra poblacional, para así reconocer con mayor claridad los cambios que se pueden presentar con el entrenamiento de fuerza en el adulto mayor, así mismo, los resultados evidencian que es posible generar cambios en porcentajes de tejido graso a través de entrenamiento en fuerza y no exclusivamente en resistencia aeróbica.

Se sugiere en próximos estudios ampliar la correlación entre fuerza explosiva y antropometría a través de este tipo de entrenamientos en población adulta mayor.

\section{Referencias}

Araya, S., Padial, P., Feriche, B., Gálvez, A., Pereira, J. \& MariscalArcas, M. (2012). Incidencia de un programa de actividad física sobre los parámetros antropométricos y la condición física en mujeres mayores de 60 años. Nutrición Hospitalaria, 27( 5 ): 1472 1479. doi:10.3305/nh.2012.27.5.5899.

Barbosa, M., J., A., P., Rodríguez, M., N., G., Hernández, H., De Valera, Y., M., Hernández, H., R., A. \& Herrera, M., H., A. (2007). Masa muscular, fuerza muscular y otros componentes de funcionalidad en adultos mayores institucionalizados de la Gran Caracas-Venezuela. Nutrición Hospitalaria, 22(5), 578-583. Recuperado de: http://scielo.isciii.es/ scielo.php? script=sci_arttext\&pid=S0212 16112007000700009\&lng=es\&tlng=es.

Bobbert, M., F., \& Soest, A. (1994). Effects of muscle strengthening on vertical jump height: a simulation study. Medicine and Science in Sports and Exercise, 26(8), 1012-1020. Recuperado de: https:// insights.ovid.com/medicine-science-sports- exercise/mespex/1994/ 08/000/effects-muscle-strengthening-vertical-jump- height/13/ 00005768

Brady, A., O, \& Straight, C., R. (2014). Muscle capacity and physical function in older women: What are the impacts of resistance training? Journal of Sport and Health Science, 3, 179-188. doi: https://doi.org/ 10.1016/j.jshs.2014.04.002
Copeland, J., L., Ashe, M., C., Biddle, S., J., Brown, W., J., Buman, M., P., Chastin, S., ... \& Owen, N. (2017). Sedentary time in older adults: a critical review of measurement, associations with health, and interventions. British Journal Sports Medicine, 51(21), 15391539. Recuperado de: http://bjsm.bmj.com/content/51/21/1539

Del Valle S., M., Prieto, J., A., Nistal, P., Martinez, P., C., \& Ruiz, L. (2014). Impacto de las estrategias de ejercicio físico en la CVRS de adultos sedentarios. Revista Internacional de Medicina y Ciencias de la Actividad Física y el Deporte, 16(64), 739-756. doi: http:// dx.doi.org/10.15366/rimcafd2016.64.008

Edholm, P., Strandberg, E., \& Kadi, F. (2017). Lower limb explosive strength capacity in elderly women: effects of resistance training and healthy diet. Journal of Applied Physiology, 123(1), 190-196. doi: https://doi.org/10.1152/japplphysiol.00924.2016

Enriquez-Reyna, M., C., Carranza, D. \& Navarro, R. (2019). Nivel de actividad física, masa y fuerza muscular de mujeres mayores de la comunidad: Diferencias por grupo etario. Retos, 35,121-125.

Foldvari, M., Clark, M., Laviolette, L., C., Bernstein, M., A., Kaliton, D., Castaneda, C., ... \& Singh, M., A., F. (2000). Association of muscle power with functional status in community-dwelling elderly women. Journals of Gerontology Series A: Biological Sciences and Medical Sciences, 55(4), M192-199. doi: https://doi.org/10.1093/ gerona/55.4.M192

Frischknecht, R. (1998). Effect of training on muscle strength and motor function in the elderly. Reproduction Nutrition Development, 38(2), 167-174. Recuperado de: https://md.edpsciences.org/articles/ md/pdf/1998/02/RND_0926-5287_1998_38_2_ART0003.pdf

García, A., J., \& Froment, F. (2018). Beneficios de la actividad física sobre la autoestima y la calidad de vida de personas mayores. Retos: nuevas tendencias en educación física, deporte y recreación, (33), 3-9. Recuperado de: https://dialnet.unirioja.es/servlet/ articulo? codigo $=6367715$

Gama, Z.,A., D., S., \& Gómez-Conesa, A. (2008). Factores de riesgo de caídas en ancianos: revisión sistemática. Revista de Saúde Pública, 42, 946-956. Recuperado de: https://www.scielosp.org/article/rsp/ 2008.v42n5/946-956/

Guillen, L., Bueno, E., Gutierrez, M., \& Guerra, J. (2018). Programa de actividad física y su incidencia en la depresión y bienestar subjetivo de adultos mayores Impact of a physical activity program on older adults' depression and subjective well-being. Retos,33,14-19. https:/ /recyt.fecyt.es/index.php/retos/article/view/49638/35594

Hall, J., A., Ochoa, P., Y., Alarcon, E., I., Moncada, J., A., Garcia, O. \& Dantas, E., H. (2017). Programa de entrenamiento de hidrogimnasia sobre las capacidades físicas de adultas mayores / Hydrogymnastics Training Program on Physical Fitness in Elderly Women. Revista Internacional de Medicina y Ciencias de la Actividad Física y el Deporte, 17 (66). 283-298

Hughes, V., A., Frontera, W., R., Wood, M., Evans, W., J., Dallal, G., E., Roubenoff, R., \& Singh, M., A., F. (2001). Longitudinal muscle strength changes in older adults: influence of muscle mass, physical activity, and health. The Journals of Gerontology Series A: Biological Sciences and Medical Sciences, 56(5), B209-B217. doi: 10.1093/ gerona/56.5.B209

Izquierdo, M., Aguado, X., Gonzalez, R., Lopez, J., L., \& Häkkinen, K. (1999). Maximal and explosive force production capacity and balance performance in men of different ages. European Journal of Applied Physiology and Occupational Physiology, 79(3), 260267. Recuperado de: https://link.springer.com/article/10.1007/ s004210050504

Juárez, S., D., Navarro, V., F., Aceña, R., R. M., González, R., J., M., Arija, B., A., \& Muñoz, Fernández, V. (2008). Relación entre la fuerza máxima en squat y acciones de salto, sprint y golpeo de balón, Revista Internacional de Ciencias del Deporte, 4(10). Recuperado de: http://www.redalyc.org/html/710/71041002/

Kemmler, W., Stengel, S., V., Weineck, J., R., Lauber, D., Kalender, W. \& Engelke, K. (2005). Exercise Effects on Menopausal Risk Factors of Early Postmenopausal Women: 3-yr Erlangen Fitness 
Osteoporosis Prevention Study Results. Medicine Science Sports Exercise, 37(2), 194-203. doi: 10.1249/ 01.MSS.0000152678.20239.76

Keogh, J., W., Kilding, A., Pidgeon, P., Ashley, L., \& Gillis, D. (2009). Physical benefits of dancing for healthy older adults: a review. Journal of aging and physical activity, 17(4), 479-500. doi: https:/ /doi.org/10.1123/japa.17.4.479

Knight, C., A., \& Kamen, G. (2001). Adaptations in muscular activation of the knee extensor muscles with strength training in young and older adults. Journal of Electromyography and Kinesiology, 11(6), 405-412. Recuperado de: https://doi.org/10.1016/S10506411(01)00023-2

Laroche, D. P., Knight, C. A., Dickie, J. L., Lussier, M., \& Roy, S. J. (2007). Explosive force and fractionated reaction time in elderly low-and high-active women. Medicine and science in sports and exercise, 39(9), 1659-1665.doi: 10.1249/mss.0b013e318074ccd9

Medina, M., A., R., Padilla, L., A., Rodríguez, E., R., A. \& Alejo, S., L., A. (2018). Aproximaciones teóricas de los efectos del entrenamiento pliométrico en agua y tierra sobre fuerza muscular y densidad mineral ósea. Revista Colombiana de Rehabilitación, 9(1), 24-31. Recuperado de: https://revistas.ecr.edu.co/index.php/RCR/article/ view/204

Ramírez, V., J., F., Da Silva, M., E., \& Lancho, A., J., L. (2007). Influencia de un programa de entrenamiento con saltos en la fuerza explosiva, la velocidad de movimiento y el equilibrio dinámico de personas mayores. Revista Española de Geriatría y Gerontología, 42(4), 218-226. doi:10.1016/S0211-139X(07)73554-0

Ramírez, V., J. F., \& León, A., H., H. (2012). Características antropométricas, funcionales y de fuerza explosiva de mujeres mayores de 50 años físicamente activas de la ciudad de Bogotá, Colombia. Revista Española de Geriatría Y Gerontología, 47(4), 148-154. doi.org/10.1016/j.regg.2012.04.005

Rendón, M., P., A., Lara, C., L. R., Hernández, J., J., Alomoto. N., M., R., Landeta, V., L., J., \& Calero M., S. (2017). Influencia de la masa grasa en el salto vertical de basquetbolistas de secundaria. Revista Cubana de Investigaciones Biomédicas, 36(1), 1-12. Recuperado de: http://scielo.sld.cu/scielo.php?script=sci_arttext\&pid=S086403002017000100015\&lng=es\&tlng=es.

Robertson, R., J., Goss, F., L., Rutkowski, J., Lenz, B., Dixon, C., Timmer, J., ... \& Andreacci, J. (2003). Concurrent Validation of the
OMNI Perceived Exertion Scale for Resistance Exercise. Medicine. Science Sports Exercise, 35(2), 333-341. doi: 10.1249/ 01.MSS.0000048831.15016.2A

Salazar, P., J., Ramírez, V., J., Chaparro, D., \& León, H. (2014). Systematic Review of the Impact of Physical Activity on Gait Disorders in the Elderly. Apunts. Educación Física Y Deportes, 4(118), 30-39. doi.org/10.5672/apunts.2014- 0983.es.(2014/ 4). 118.03

Sandoval, C., C., Camargo, M., González, O., D., \& Vélez, R., Y. (2007). Programa de ejercicio físico para los adultos mayores del Club Nueva Vida de la ciudad de Tunja. Revista Ciencia de la Salud, 5(2), 60-71. Recuperado de: http://www.scielo.org.co/pdf/recis/ v5n2/v5n2a7.pdf

Sánchez, I., A. (2009) Entrenamiento de la fuerza muscular como coadyuvante en la disminución del riesgo cardiovascular: una revisión sistemática. Revista Colombiana de Cardio. Revista Colombiana de Cardiologia, 16(6), 239-248.

Stengel, S., V., Kemmler, W., Pintag, R., Beeskow, C., Weineck, J., Lauber, D., ... Engelke, K. (2005). Power training is more effective than strength training for maintaining bone mineral density in postmenopausal women. Journal of Applied Physiology, 99(1). Recuperado de: http://jap.physiology.org/content/99/1/181.short Vaquero-Cristóbol, R., Martínez, I., Alacid, F., Ros, E. (2015). Efectos de la lateralidad sobre la flexibilidad, la fuerza-resistencia y el equilibrio en mujeres mayores activas. Retos, 27, 127-130. Recuperado de: https://recyt.fecyt.es/index.php/retos/article/view/34362/18542

Vidarte, C., J., A., Quintero, C., V., M. \& Herazo, B., Y. H. (2012). Efectos del ejercicio físico en la condición física funcional y la estabilidad en adultos mayores. Revista hacia la promoción de la salud, 17(2), 79-90. Recuperado de: http://www.scielo.org.co/pdf/ hpsal/v17n2/v17n2a06.pdf

Villareal, M., A., Moncada, J., Gallegos, J., J. \& Ruiz, F. (2016). El efecto de un programa de ejercicios basado en Pilates sobre el estado de ánimo en adultos mayores mexicanos. Retos, 30,106109. Recuperado de: ttps://recyt.fecyt.es/index.php/retos/article/ view/49543/30460

Zillikens, M. C., Demissie, S., Hsu, Y. H., Yerges-Armstrong, L. M., Chou, W. C., Stolk, L., ... \& Kutalik, Z. (2017). Large meta-analysis of genome-wide association studies identifies five loci for lean body mass. Nature Communications, 8(1), 80.

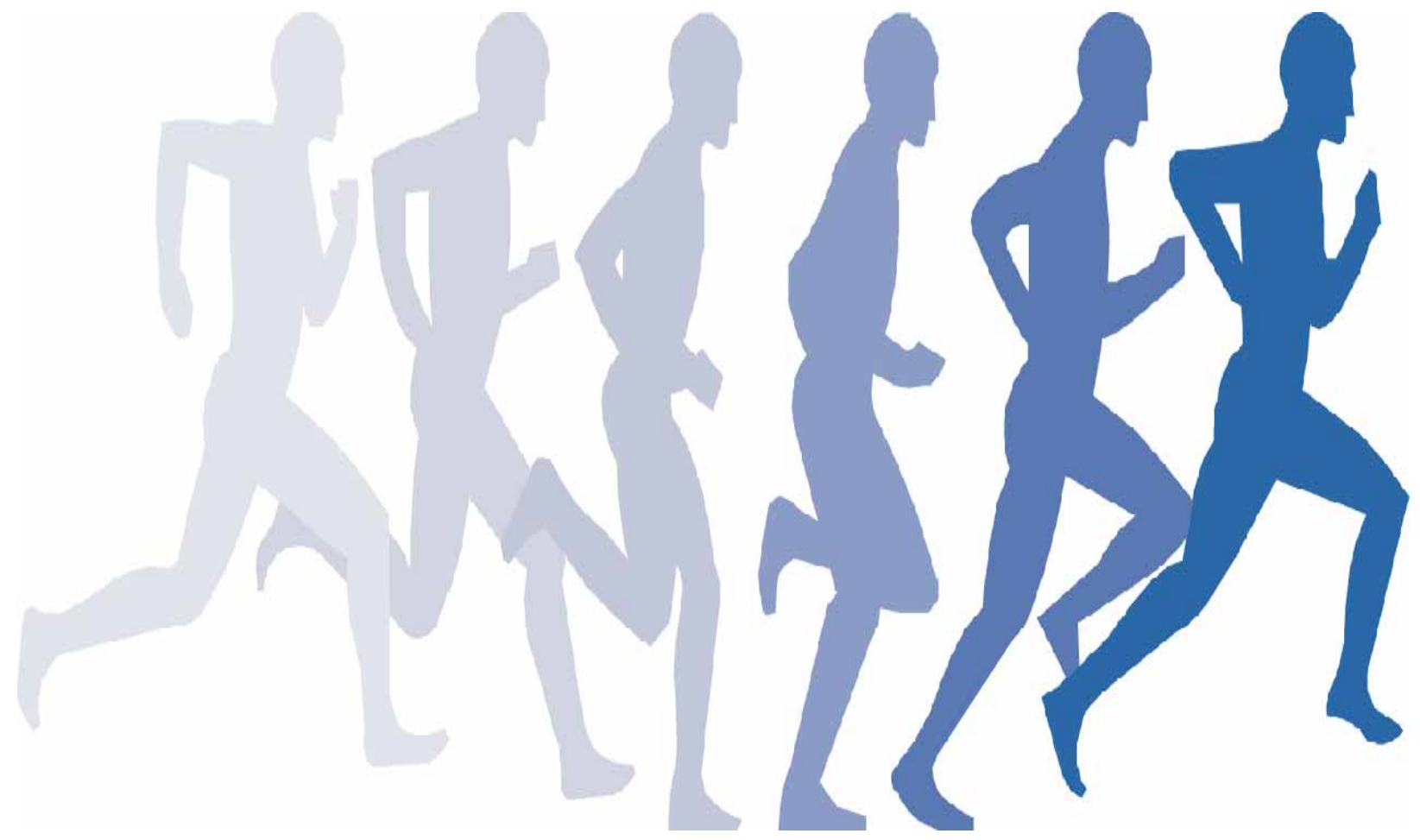

